\title{
MAP Attitude Control System Design and Flight Performance
}

\author{
S. F. Andrews, I. R. O'Donnell, Jr.. Ph. D. \\ Flight Dynamics Analysis Branch \\ NASA Goddard Space Flight Center \\ Greenbelt, MD 2077J USA
}

\section{Summary}

The Microwave Anisotropy Probe (MAP) is a follow-on to the Differential Microwave Radiometer (DMR) instrument on the Cosm:c Background Explorer (COBE) spacecraft. To make a full-sky map of cosmic microwave background luctuations. a combination fast spin and slow precession motion will be used that will cover the entire celestial sphere in six months. The spin rate should be an order of magnitude higher than the precession rate, and each rate should be tightly controlled. The sunline angle should be $22.5^{\circ}+1-0.25^{\circ}$. Sufficient attitude knowledge must be provided to yield instrument pointing to a standard deviation of 1.3 arc-ninutes RSS three axes. In addition, the spacecraft must be able to acquire and hold the sunline at inital accuisition, and in the event of a failure. Finally, the spacecraft must be able to slew to the proper burn orientations and to the proper off-sunline attitude to start the compound spin.

The design and flight performance of the Attitude Control System on MAP that meets these requirements will be discussed.

There are also requirements for performing orbit adjust and momentum unloading maneuvers, as well as requirements for an independen' safehold, but those are beyond the scope of this paper. Also, trajectoryrelated requirements will not be discussed unless they impact directly on the system performance or design. The RWA-based control modes and attitude determination system that reside in the Mongoose $\mathrm{V}$ processor will be discussed.

MAP uses three right-handed, o thonormal coordinate systems. The Geocentric Inertial frame (GCI) is an Earth-centered frame in which the $x_{I}$ axis points to the vernal equinox, the $z_{1}$ axis points to the North Celestial Pole (parallel to the Earth's spin axis), and $y_{1}=z_{1} \times x_{1}$. The Rotating Sun Referenced frame (RSR) is a spacecraft-centered frame in which $z_{R}$ axis points to the Sun, $x_{R}=z_{R} \times z_{1}$, and $y_{R}=z_{R} \times x_{R}$. The RSR frame rotates at approximately $1^{c} /$ day with respect to the GCI frame. The body frame is centered at the spacecraft center of mass with the $z_{\mathrm{B}}$ axis parallel to the spacecraft centerline, directed from the instrument to the solar arrays. The $y_{B}$ axis is normal to the instrument radiator faces, and $\mathrm{x}_{\mathrm{B}}=\mathrm{y}_{\mathrm{B}} \times \mathrm{z}_{\mathrm{B}}$.

The attitude sensors employed ty MAP are an Inertial Rate Unit (IRU), an Autonomous Star Tracker (AST), a two-axis Digital Sun Sensor (DSS), and twelve Coarse Sun Sensors (CSSs). The spacecraft attitude will be controlled by three Reaction Wheel Assemblies (RWAs), and six Reaction Engine Modules (REMs). or thrusters. The ACS control algorithms are implemented in software in the Mongoose V processor. Sensor data acquisition, actuator command generation, and an independent safehold algorithm are in the At:itude Control Electronics Remote Services Node (ACE RSN).

\section{ACS Operational Modes}

There are six ACS operational nodes: Inertial. Observing, Delta V, Delta H, Sun Acquisition, and Safehold. The first five modes are implemented in the Mongoose, while the Safehold Mode resides in the ACE. Anomalous behavior can result in autonomous transitions from any other mode to Safehold Mode 
or Sun Acquisition Mode, even hough these transitions are not shown explicitly. The thruster controlled modes, Delta V and Delta H, will not be discussed in this paper, nor will the Safehold Modes.

Sun Acquisition Mode acquires and maintains a thermally-safe power-positive orientation for the spacecraft. At launch, the MAP ACS was in Sun Acquisition Mode, which was used for initial acquisition. Normal exit from Sun Acquisition Mode is to either Delta H or Inertial Mode. depending on the residual spacecraft spin rate. It the rates exceed those that can be handled by the RWA-based Sun Acquisition Mode, the REM-bas.ed Delta H Mode is entered to reduce the rates to an acceptable level, after which the spacecraft returns to Sun Acquisition Mode. Transition to Inertial Mode can be commanded after the Sun has be en acquired. Transition to the Mongoose control modes from the ACE Safehold Mode is through Sun Acquisition Mode.

Inertial Mode acts as a staging mode between the other operations of the spacecraft; all Mongoose modes other than Delta V have the capability to enter this mode. Inertial Mode can either hold the spacecraft in an inertially-fixed orientation or slew the spacecraft between two different orientations. This is an RWAand IRU-based mode, with DSS and AST measurements used in an onboard Extended Kalman Filter (EKF) to update the gyro bias and quaternion error estimates. Normal exits from Inertial Mode are by ground command only.

Observing Mode is used for science operations. The RWAs provide the angular momentum that maintains a near-zero system momentum and the torques that maintain both the $22.5^{\circ}$ angle between the spin axis and Sun line and the desired sky-scan rates. This is also an IRU-based mode, using the same EKF as Inertial Mode. Normal transitions into or out of this mode are via Inertial Mode.

ACS Design and Performance

Each of the RWA-controlled operational modes of the MAP ACS will be discussed below. Each mode discussion will give the sensors and actuators used in that mode, discuss the control algorithm, and give an example of the performance of the mode.

Sun Acquisition Mode

Sun Acquisition Mode slews the spacecraft to a power-positive orientation within $25^{\circ}$ of the sun. The attitude error signal is calculated from differencing the CSS eye measurements, and crossing that vector with the desired sun vector. That signal is limitted, and multiplied by the proportional control gain. The rate error signal is the body rate measured by the IRUs. The rate error is multiplied by the spacecraft inertia and then multiplied by a derivative control gain. The output of this PD control is the RWA control torques that get sent to the RWA to control the spacecraft.

Figure 1 shows the inital sun acquisition after spacecraft separation from the third stage of the launch vehicle. 


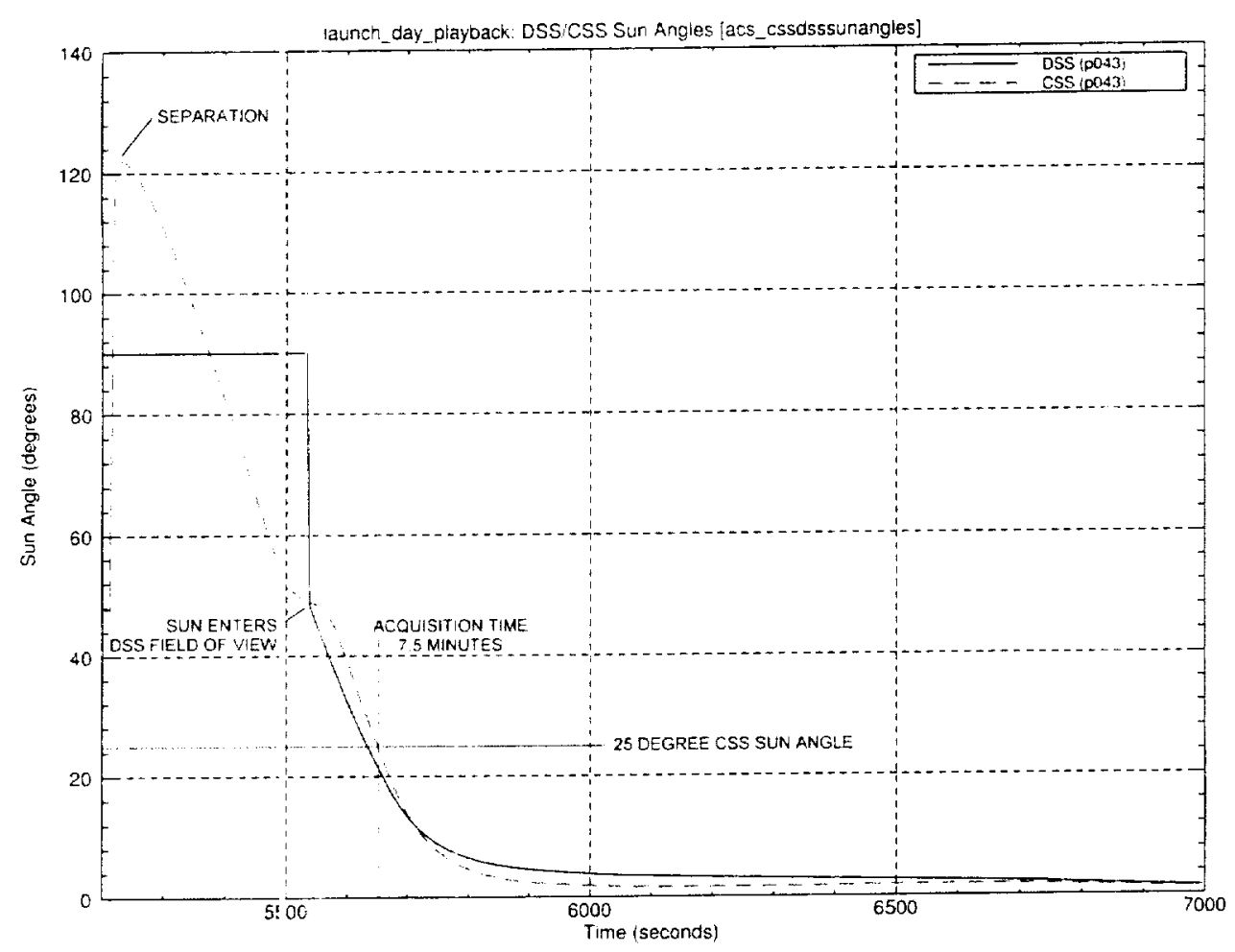

Fi izure 1 CSS and DSS Sun Angle at Separation

Inertial Mode

Inertial Mode can either hold thə spacecraft in an inertially-fixed orientation or slew the spacecraft to a different orientation. The desired orientation is ground-commanded as a desired GCI-to-body quaternion $q_{c}[1,2]$; a slew will be executed if this is not close to the current spacecraft orientation. The attitude control is by RWA torques, which are computed by a proportional-integral-derivative (PID) controller in terms of attitude and rate errors. The attitude errors are expressed as the vector part $\vec{q}_{e}$ of an error quaternion, which is given as th? quotient of the commanded quaternion and an estimated quaternion $\hat{q}$ :

$$
q_{\mathrm{e}}= \pm q_{\mathrm{c}} \otimes \hat{q}^{-1}
$$

In this and the following we use the quaternion product convention of [2,3] rather than that of [1], so that the order of quaternion multiplication is the same as that of the corresponding direction cosine matrices. The sign in the equation above is chosen so that the scalar component of $q_{e}$, which should have magnitude close to unity for small pointing errors, is positive.

The estimated quaternion is computed by an onboard Extended Kalman Filter (EKF), with IRU, AST, and DSS measurements as input [3-5]. This is similar to EKFs employed on several previous missions, except that the AST produces a measured attitude quaternion rather than merely observed star vectors. This simplifies the Mongoose computations by removing the burden of star identification and the necessity to carry an onboard star catalog. In addition, both the DSS measurements and the AST measurements are back-propagated to the IRU meatsurement time to reduce the effect of timing errors. especially in Observing Mode. 
Since the desired rates in Inertial Mode are zero, the rate error vector $\bar{\omega}_{c}$ is the negative of the body rate vector $\bar{\omega}_{\mathrm{B} 1}$ measured by the IRI $\mathrm{I}$ :

$$
\vec{\omega}_{e}=-\vec{\omega}_{\mathrm{BI}}
$$

\section{Observing Mode}

Observing Mode is used for science operations to maintain the $22.5^{\circ}$ angle between the spin axis and antiSun line and the desired sky-scan rates as shown in Figure 2. Observing Mode differs from Inertial Mode in that the commanded quaternion is time-varying and the commanded rates are non-zero. There are also a commanded acceleration and a gyroscopic torque, which are used in a feedforward loop to eliminate an attitude hangoff. The commanded inertial quaternion is computed as the product of an RSR-to-body and a GCI-to-RSR quaternion:

$$
q_{\mathrm{BI}}=q_{\mathrm{BR}} \otimes q_{\mathrm{RI}}
$$

The GCI-to-RSR quaternion is computed onboard from ephemeris models. The desired RSR-to-body attitude is more conveniently expressed in terms of 3-1-3 Euler angles [1.2] than in terms of a quaternion. The commanded values of the three Euler rates are those that give the desired scan rates:

$$
\begin{aligned}
& \dot{\phi}_{c}=1 \mathrm{rev} / \mathrm{hour}=0.001745 \mathrm{rad} / \mathrm{sec} \\
& \dot{\theta}_{c}=0 \\
& \dot{\psi}_{c}=0.464 \mathrm{rpm}=0.04859 \mathrm{rad} / \mathrm{sec}
\end{aligned}
$$

The commanded values of the tlıree Euler angles are then given by:

$$
\begin{aligned}
& \phi_{r}=\phi_{0}+\int_{t_{0}}^{t} \dot{\phi}_{r} d t \\
& \theta_{r}=225^{\circ}=0.3927 \mathrm{rad} \\
& \psi_{r}=\psi_{0}+\int_{t_{0}}^{t} \dot{\psi}_{r} d t
\end{aligned}
$$

where $\phi_{0}$ and $\psi_{0}$ are set by the nitial state and $\theta_{r}$ by the desired Sun angle. These 3-1-3 Euler angles are converted to the commanded R: $\mathrm{R}$-to-body quaternion $q_{\mathrm{BR}}$ by the standard equations [1,2].

The rate error vector $\vec{\omega}_{e}$ is the difference between the body rate vector $\vec{\omega}_{\mathrm{BI}}$ measured by the IRU and a commanded body rate vector $\vec{\omega}_{i}$ :

$$
\vec{\omega}_{\iota}=\vec{\omega}_{B I}-\vec{\omega}_{c}
$$

The commanded body rate vector $\bar{\omega}_{,}$is computed from the commanded Euler angles and rates by the standard equations shown below: $[7,8]$.

$$
\bar{\omega}_{r}=\left[\begin{array}{ccc}
\sin \theta_{r} \sin \psi_{c} & \cos \psi_{c} & 0 \\
\sin \theta_{r} \cos \psi_{c} & -\sin \psi_{c} & 0 \\
\cos \theta_{r} & 0 & 1
\end{array}\right]\left[\begin{array}{c}
\dot{\phi}_{r} \\
\dot{\theta}_{r} \\
\dot{\psi}_{r}
\end{array}\right]
$$


The additional acceleration feecforward term is the acceleration the spacecraft needs to follow the commanded attitude and Euler rates, and is derived by differentiating the equation for $\vec{\omega}_{c}$ with respect to time. Since $\dot{\phi}_{r}, \dot{\psi}_{c}$, and $\theta_{c}$ are all constant, the commanded acceleration is:

$$
\dot{\bar{\omega}}_{c}=\dot{\psi}_{c} \phi_{c} \sin \theta_{c}\left[\begin{array}{c}
\cos \psi_{c} \\
-\sin \psi_{c} \\
0
\end{array}\right]
$$

The gyroscopic feedforward torque, used to move the system momentum inthe body frame, is

$$
\vec{T}_{g}=\vec{\omega}_{c} \times \vec{H}_{s y}
$$

These terms are added to the co nmanded acceleration to give the control torque:

$$
\vec{T}=J\left[k_{d} \vec{\omega}_{c}+k_{p}\left(2 \vec{q}_{e}\right)+\dot{\vec{\omega}}_{r}\right]+\vec{T}_{g}
$$

Observing Mode performance i:; shown in Figure 2.

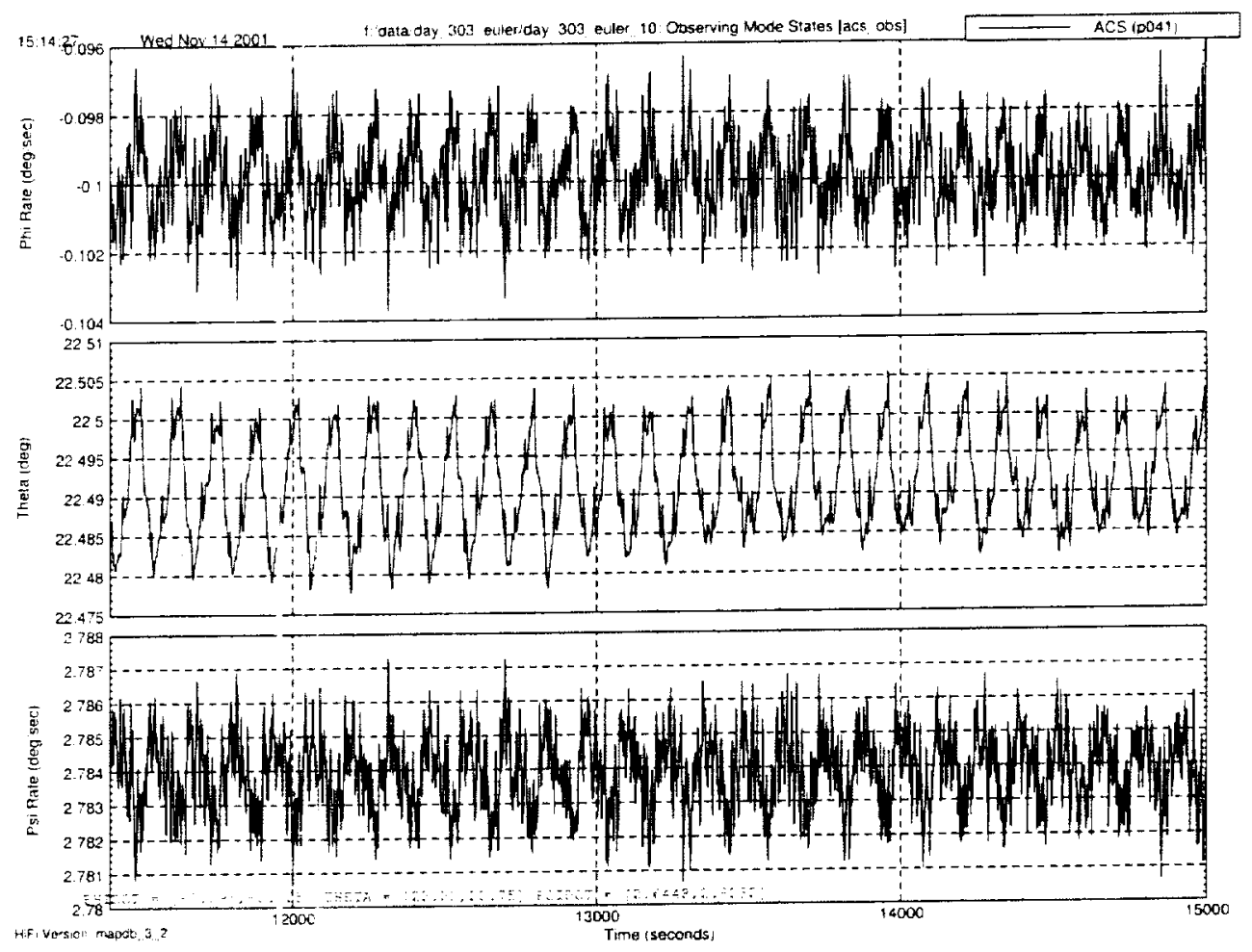

Figure 2 Post-Calibration Observing Mode Performance 
Kalman Filter

The MAP Kalman filter is an Extended Kalman Filter that uses AST and DSS measurements to update the gyro-propagated attitude and to update the estimated gyro bias. The spacecraft does not use the estimated attitude while in Sun Acquisition Mode, since it is flying off the CSS measurements. The EKF is used to provide an attitude estimate durng Inertial and Observing Modes. There are States, or submodes, within each control mode that are used to determine whether or not the filter will use the measurements to try to update the attitude and bias. In general, if the control mode produces accelerations on the body, the Kalman filter is set to propagate the covariance matrix, and the estimated attitude and gyro bias are not updated. In Inertial Hold and Observing Scan/Hold, the Kalman filter is used to update the estimates. The measurement residuals are $\mathrm{a}$ good indication of how well the EKF is estimating the spacecraft attitude. Figures 3 and 4 show a typical one-hour period in Observing Mode at L2. The short-period oscillations are due to inaccuracies in the first-order attitude propagator used onboard, and the longer period oscillations are due to snall residual misalignments between the AST and the DSS, showing up at the precession period.

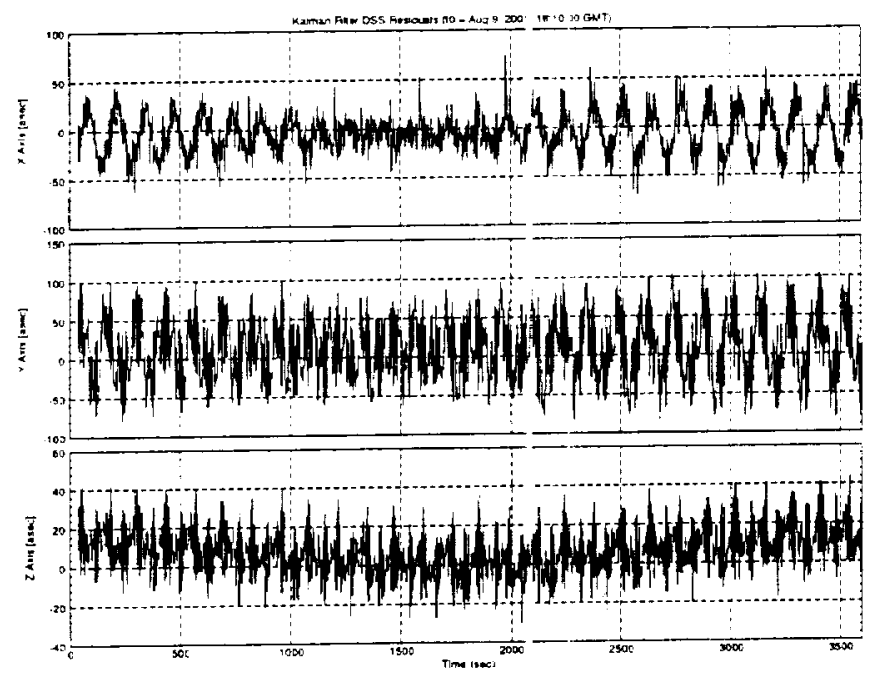

Figure 3 DSS Residuals

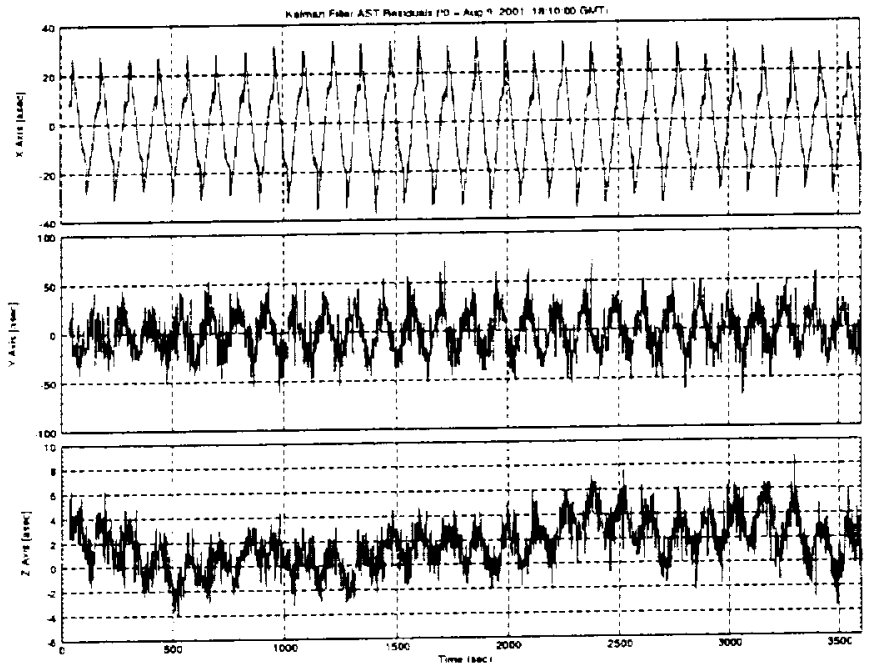

Figure 4 AST Residuals

OLTLINE

1. MAP Mission Overview

1.1 Science Objectives

1.2 Requirements

1.3 Scope of paper

2. Control Modes

2.1 Sun Acquisition Mode

2.1.1 design

2.1.2 performance

2.2 Inertial Mode

2.2.1 design

2.2.2 performance

2.3 Observing Mode

2.3.1 design

2.3.2 performance

3. Attitude Determination 
3.1 Sun Acquisition

3.2 Extended Kalman Filter

3.2.1 Basic design

3.2.2 Modifications for Quaternion Out AST

3.2.3 Operations

3.2.4 Performance in Osserving Mode

4. Conclusion

[1] Wertz, J. R., ed., Spacecraft Ittitude Determination and Control. D. Reidel. (1978).

[2] Shuster, M. D., Journal of the Astronautical Sciences, vol. 41, p. 439 (1993).

[3] Lefferts. E. J., Markley, F. L., and Shuster, M. D., Journal of Guidance, Control, and Dynamics. vol. 5, p. 417 (1982).

[4] Murrell, J. W., AIAA Paper :8-1248, AIAA Guidance, Navigation, and Control Conference. Palo Alto. CA (1978).

[5] Bauer, F. H., Femiano, M. D.. and Mosier, G. E., AIAA Paper 92-4334-CP. AlAA Guidance, Navigation. and Control Conference, Hilton Head, SC (1992).

[6] Mortensen, R. E., International cournal of Control, vol. 8. p. 297 (1968).

[7] Wie. B., and Barba, P. M., Je urnal of Guidance, Control, and Dynamics, vol. 8, p. 360 (1985). 